\title{
III. On certain Points connected with scale Order in the Case of the Correlation of two characters which for some arrangement gtve a Inear Regresaton Ine.
}

\author{
BY KARL PEARSON, F.RS.
}

In a recent memoir on contingency , I have considered the problem of what altarations can be made in scale onder withort sensibly modifying the value of the correlation. The problem as I there state it is as follows: To find urdor what other condition than normal correlation small changes in the order of grouping will not affect the value of the correlation (p. 19). The wording requires some explanation. If for any arrangement of the scales of the two variables there be normal correlation, then my memoir shows that the method of contingency gives the value of the correlation, even if the order of the scales be any whatever, in fact if the normal correlation order be sbsolutely unknown. Of course, if we proceed in any such case by the usual product method of determining the correlation we shall reach absolntely different results when the scale order of grouping is largely changed. My object in stating the above problem was to determine, if possible, whether any and if so what changes in the scale orders would not sensibly modify the correlation, when we still endearoured to determine it, not by contingency, but by the method of products. The conclusion I came to was as follows-that with any distribution with linear regression "small changes (i.e such that the sum of their squares may be neglected as compared with the square of mean or standard deviation) may be made in the order of grouping without affecting the correlation coefficient" (p. 35). I think this conclusion is quite sound, and deserves further consideration. Although in the statement of the proposition I have used the word "small changes" in scale order (p. 19) and in the summary of my memoir (p. 35) stated what is to be understood by small, in this case, I think, as Mr G. U. Yule points out to me, that the wording on p. 20 is too unguarded, if the reader has not been sufficiently impressed with the wording on p. 19, or reached the summary on p: 35 . It will not be without value possibly to give the actual algebraical result on which the statement on p. 35 is based, for it has some importance for the general philosophical ides of correlation.

Let $x$ and $y$ represent the two variable characters and let $u d x$ be the frequency of the character between $x$ and $x+2 x$; vyy that of the character between $y$ and $y+\partial y$; $u$ and $v$ being functions of $x$ and $y$ respectively and the distribution of the frequencies being of any nature. Now suppose the array $v_{8} \delta y_{0}$ of. frequency between $y_{0}$ and $y_{0}+\delta y_{0}$ to be bodily interchanged in position with the array $v_{,} \delta y_{\gamma}$ between $y_{\gamma}$ and $y_{\gamma}+\delta y_{\gamma}$. Let $N$ be the total frequency, and suppose the mean $\bar{y}$ to become $\bar{y}+\delta \bar{y}$, the standard deviation $\sigma_{y}$ of the $y$ character to become $\sigma_{y}+\delta \sigma_{r}$ Then wo have :

or

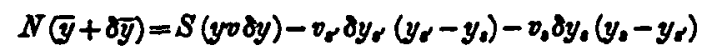

$$
\begin{aligned}
& \delta \bar{y}=\left(y_{0}-y_{p}\right) \frac{\left(v_{p} \delta y_{p}-v_{s} \delta y_{0}\right)}{N}
\end{aligned}
$$

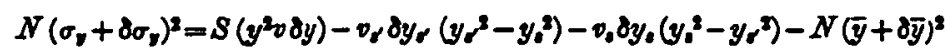

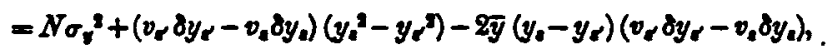

$$
\begin{aligned}
& N\left(\delta \sigma_{v}\right)^{2}+2 N \sigma_{\gamma} \delta \sigma_{\nu}=\left(v_{\alpha} \delta y_{\alpha}-v_{0} \delta y_{0}\right)\left(y_{0}-y_{\sigma^{\prime}}\right)\left(y_{0}-\bar{y}+y_{\gamma}-\bar{y}\right) .
\end{aligned}
$$

- "Mathematical Contribations to the Theory of Erolution, III. On the Theory of Contingency and its Relation to Association and Normal Correlstion." Drapere' Researeh Memoirs (Dolan and Co. London). 
Hence we seo that $\delta \sigma_{y}$ is small, if the frequencies of interchanged subgroups are stall as compared with $N$ and acoordingly:

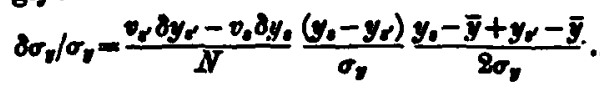

We now turn to the change in the product-moment.

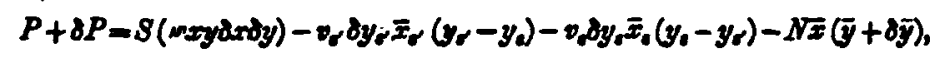

where woxy is the total frequency of individuals, with characters between $x$ and $x+d x$ and $y$ and $y+\delta y$ and $\bar{x}_{a}$ and $\bar{x}_{8}$ are the means of the arrays corresponding to $y_{0}$ and $y_{\gamma}$. But $P=S($ roxy $8 x \delta y)-N \overline{x y}$, henco:

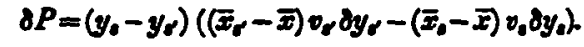

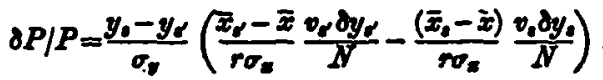

Now if $r$ be the corralation bafore and $r+$ or after a change is made, we bave, since $r=P /\left(N \sigma_{z} \sigma_{v}\right)$

$$
\frac{\partial r}{r}=\frac{\partial P}{P}-\frac{\delta \sigma_{z}}{\sigma_{z}}-\frac{\partial \sigma_{y}}{\sigma_{y}}
$$

Now we hare supposed at present no change to be made in the 2 ; thus we may treat $\delta \sigma_{x}$ as zero, and tusing (ii) and (iii) we have, rearranging :

$$
\begin{aligned}
& \frac{\partial r}{r}=\frac{y_{0}-y_{g}}{r \sigma_{y} \sigma_{z}}\left[\frac{v_{p} \partial y_{\gamma}}{N}\left\{\bar{x}_{p}-\bar{x}-\frac{r \sigma_{z}}{\sigma_{z}}\left(y_{p}-\bar{y}\right)\right\}-\frac{v_{p} \partial y_{0}}{N}\left\{\bar{x}_{0}-\bar{x}-\frac{r \sigma_{z}}{\sigma_{y}}\left(y_{0}-\bar{y}\right)\right\}\right] \\
& -\frac{\left(y_{2}-y_{\gamma}\right)^{2}}{2 \sigma_{\nu}^{2}} \frac{v_{\gamma} \delta y_{\gamma}+v_{s} \delta y_{0}}{N}
\end{aligned}
$$

Now suppose the regression to be originally linesr, then we bave $\bar{x}_{2}-\bar{x}=\frac{r \sigma_{x}}{\sigma_{y}}\left(y_{0}-\bar{y}\right)$ not only for and 8 but for all values of a whatever. In other wonds the whole series of tarms in square brackets vanishes and summing for all pairs of interchanges:

$$
\frac{\Delta r}{r}=-\frac{S\left(y_{0}-y_{p}\right)^{2}\left(v_{p} \delta y_{\rho}+v_{0} \delta y_{0}\right)}{2 N \sigma_{\gamma}^{2}}
$$

If we make similar interchanges of $x_{p}$ and $x_{p}-$ we can show that :

$$
\begin{aligned}
& \frac{\partial r}{r}=-\frac{S\left(y_{0}-y_{p}\right)^{2}\left(v_{p} \partial y_{p}+v_{0} \partial y_{p}\right)}{2 N \sigma_{p}^{2}}-\frac{s\left(x_{p}-x_{p}\right)^{2}\left(u_{p} d x_{p}+u_{p} d x_{p}\right)}{2 N \sigma_{p}^{2}} \\
& +\frac{S^{\prime \prime}\left(y_{0}-y_{p}\right)\left(x_{p}-x_{p}\right)\left(w_{1} \partial x_{p} \partial y_{p}-w_{2} \partial x_{p} \partial y_{0}-v_{p} \partial x_{p} \partial y_{p}+i_{q} \partial x_{p} \partial y_{p}\right)}{N r \sigma_{z} \sigma_{p}} \ldots \text {... (vi) bia }
\end{aligned}
$$

Here $S$ denotes a summation or integration for all possible interchanges of the $y$ arrays, i.a. aay, columns of the correlation table; and $S^{\prime}$ denotes a like summation for all possible interchangea of the $\dot{x}$-arrays, say the rows of the table. $S^{\prime \prime \prime}$ is a summation involving the frequency at all points where iuterchanged rows and columns cross Of course this result assumes that the units of grouping of both characters are 80 "fine" that the squares of the ratios of the array frequencies to the total frequency are negligible.

We may now draw some interesting conclusions from (vi). Suppose the material to be auch that the correlation is linear under some arrangement. Then for slight interchanges the squares and products of the interchanges are negligible and $8 r$ will be zero. Thus, $r$ being positive, we

- The reader will ind a verifcestlon of this formuls artaing from writing (i) the correlation teble with its colamna inverted, then $8 r / r=-2$, and (ii) again in addition with its rows written backwards, in thir $8 r / r=0$. In (i) the first term only remaing and its nomerator $=4 N_{\sigma y}{ }^{2}$. In the cocond cace the namerators of the three terms are respectively $4 \mathrm{N \sigma}_{z}{ }^{2}, 4 \mathrm{No}_{z}^{2}$ and $4 \mathrm{Nr}_{z} \sigma_{r}$

Biometrika v 
800 from ( $v$ i) that $r$ is an absolute maximum. Clearly $8 r / r$ is always negative even for interchanges between arrajs at considerable distances $O r$, we conclude that if there be one arrangoment of the material for which the regression line is linear, then any interchanges, however extonsive, will reduce the value of the correlstion as calculated by the product moment method. This conception of the linear regression line as giving the arrangement with the maximum degree of correlation appears of considerable philosophical interest. It amounts practically to much the anwe thing as saying that if we have a fine clasification, we shall get the maximum of correlation by arranging the arrags so that the means of the arrays fall as closely as possible on o line.

Further, if the mean square of the interchanges, Le. the expression

$$
\frac{S\left(y_{0}-y_{\rho}\right)^{2}\left(v_{0}+y_{\rho}+v_{0} \delta y_{0}\right)}{2 N}
$$

be small as compared with the standard deviation squared, i.e. $\sigma_{y}^{3}$, then the change or will not be sensible. In other words mall changes in the scale ordering, not confined to adjacent or eren to two arrays, will not sensibly modify the currelation as found by the product moment method.

Lastly, considering the proof of (vi) we see that no portion of the investigation is dependent on the whole of the ove $y$-array being interchanged with the whole of another. We may consider $v, \delta y$, and $v, \delta y$, as only portions of the total array-to be taken, however, proportionately from all its constituents. Now let $V_{s} \delta y_{0}$ and $V_{s} \delta y_{0}$ denote the whole of the frequency of the two arrays, and write the first array $V_{0} \delta y_{0}+\frac{1}{2} m-\frac{1}{2} m$ and the second array $V_{r} \delta y_{r}-\frac{1}{2} m+\frac{1}{2} m$. Now transfer the $-\frac{1}{2} m$ of the first array to the position of the second and the $+\frac{1}{2} m$ of the second to

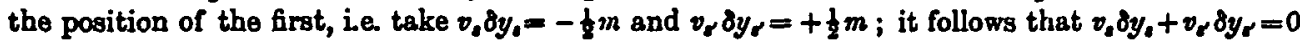
and the two arrays are

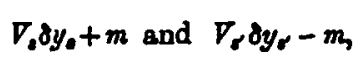

i.e exactly the values they would have had if a portion of the second array drawn at random from all its sub-groups hud been inscribed in the same sub-groups of the first array. But in this caso we see since $v_{0} \delta y_{0}+v_{r} \delta y_{\gamma}=0$, that (vi) will give us absolutely $\delta r=0$, or there will be no change in the correlation. This result seems of considerable value. Suppose the regression linear, and sne character, $x$ say, easily measured or known; then if a number $m$ of individuals which ought to fall into a given class of $y$, be shifted by oversight or error of judgment into a second erroneous class of $y$, this will not sensibly affect the correlation, if $\nu$ being the total frequency, the square of the ratio $m / N$ is negligible, as compared with its first power. Thus suppose in correlating age with hair tint, the first character being accurately known, an observer were to place his series of contributory observations of hair tint in the wrong group, say in one of the brown reds instead of pure browns, this would not sensibly modify the resultiug correlation. The fact that the error would not produce a modification is not in the first place due to the possible smallness of the misplaced group. The product moment is changed and the standard deviation is also modified, but the modification of the correlation depends on such mamer on the changes of these two, that they act in opposite senses and cancel the modification, provided the original regression was strictly linear.

While not desiring to encourage carelessness in observing or tabling or in the formation of scale orders without due consideration, still the results of this nuie seen to indicate that in many cases absolute unanimity of judgment in classifying or great stress on small details of scale grouping are not needful in order to reach sensibly idcntical values of the correlation. This view coincides with my actual and not-unique experience, when having been in greve doubt as to where 30 or 40 individuals were to be placed, I put them first in one category and then in a second, only to find out that the correlation worked out with the group first in one and then in the other category was sensibly identical. The theorems developed in this note seen to explain this stability-when we use not contingency but product moment methods, and suppose the regression ultimately linear. 